ELECTRONIC RESEARCH ANNOUNCEMENTS OF THE AMERICAN MATHEMATICAL SOCIETY

Volume 11, Pages 95-102 (December 15, 2005)

S $1079-6762(05) 00152-6$

\title{
HUREWICZ-LIKE TESTS FOR BOREL SUBSETS OF THE PLANE
}

\author{
DOMINIQUE LECOMTE
}

(Communicated by Alexander Kechris)

\begin{abstract}
Let $\xi \geq 1$ be a countable ordinal. We study the Borel subsets of the plane that can be made $\boldsymbol{\Pi}_{\xi}^{0}$ by refining the Polish topology on the real line. These sets are called potentially $\boldsymbol{\Pi}_{\xi}^{0}$. We give a Hurewicz-like test to recognize potentially $\boldsymbol{\Pi}_{\xi}^{0}$ sets.
\end{abstract}

\section{Preliminaries in Dimension one}

Let us recall some results in dimension one before studying Borel subsets of the plane. In descriptive set theory, a standard way to see that a set is complicated is to note that it is more complicated than a well-known example. For instance, we have the following result (see $[\mathrm{SR}]$ ):

Theorem 1 (Hurewicz). Let $P_{f}:=\left\{\alpha \in 2^{\mathbb{N}} / \exists n \in \mathbb{N} \quad \forall m \geq n \quad \alpha(m)=0\right\}$, X be a Polish space, and $A$ a Borel subset of $X$. Then exactly one of the following holds:

(a) The set $A$ is $\Pi_{2}^{0}(X)$.

(b) There is $u: 2^{\mathbb{N}} \rightarrow X$ continuous and one-to-one with $P_{f}=u^{-1}(A)$.

This result has been generalized to the other Baire classes (see [Lo-SR]). We state this generalization in two parts:

Theorem 2 (Louveau-Saint Raymond). Let $\xi<\aleph_{1}, A_{1+\xi} \in \boldsymbol{\Sigma}_{1+\xi}^{0}\left(2^{\mathbb{N}}\right)$, X be a Polish space, and $A, B$ disjoint analytic subsets of $X$. One of the following holds:

(a) The set $A$ is separable from $B$ by a $\Pi_{1+\xi}^{0}(X)$ set.

(b) There is $u: 2^{\mathbb{N}} \rightarrow X$ continuous with $A_{1+\xi} \subseteq u^{-1}(A)$ and $2^{\mathbb{N}} \backslash A_{1+\xi} \subseteq u^{-1}(B)$.

If we moreover assume that $A_{1+\xi} \notin \mathbf{\Pi}_{1+\xi}^{0}$, then this is a dichotomy (in this case, and if $\xi \geq 2$, we can have $u$ one-to-one).

Theorem 3. There is a concrete example of $A_{1+\xi} \in \boldsymbol{\Sigma}_{1+\xi}^{0}\left(2^{\mathbb{N}}\right) \backslash \boldsymbol{\Pi}_{1+\xi}^{0}\left(2^{\mathbb{N}}\right)$, for $\xi<\aleph_{1}$.

If we replace $P_{f}$ (resp., $\boldsymbol{\Pi}_{2}^{0}$ ) with the set $A_{1+\xi}$ given by Theorem 3 (resp., $\boldsymbol{\Pi}_{1+\xi}^{0}$ ), we get the generalization of Theorem 1 for $\xi \geq 2$. We state this generalization in two parts for the following reasons:

Received by the editors July 29, 2005.

2000 Mathematics Subject Classification. Primary 03E15; Secondary 54H05.

Key words and phrases. Potentially, Baire classes, reduction, Hurewicz's Theorem.

(C)2005 American Mathematical Society Reverts to public domain 28 years from publication 
- Theorem 2 is valid for any $A_{1+\xi} \in \boldsymbol{\Sigma}_{1+\xi}^{0}\left(2^{\mathbb{N}}\right)$, and Theorem 1 is of the form "There is a typical example such that ...".

- We will meet again a statement in two parts, in dimension two.

\section{Results With the Usual notions of Reduction}

Let us consider the case of dimension two. The usual notion of comparison for Borel equivalence relations is the Borel reducibility quasi-order (recall that a quasiorder is a reflexive and transitive relation). This means that if $X$ (resp., $Y$ ) is a Polish space, and $E$ (resp., $F$ ) a Borel equivalence relation on $X$ (resp., $Y$ ), then $E \leq_{B} F \Leftrightarrow \exists u: X \rightarrow Y$ Borel with $E=(u \times u)^{-1}(F)$.

Note that this makes sense even if $E, F$ are not equivalence relations. We will study a natural invariant for $\leq_{B}$. Recall the following (see $[\mathrm{K}]$ ):

Theorem 4 (Kuratowski). Let $X$ be a Polish space, and $\left(B_{n}\right)$ a sequence of Borel subsets of $X$. Then there is a finer Polish topology $\sigma$ on $X$ (and thus having the same Borel sets) making the $B_{n}$ 's clopen.

In particular, if $u: X \rightarrow Y$ is Borel, there is $\sigma$ such that $u:[X, \sigma] \rightarrow Y$ is continuous. If moreover $E=(u \times u)^{-1}(F)$ and $F$ is in some Baire class $\Gamma$, then $E \in \Gamma\left([X, \sigma]^{2}\right)$. This leads to Definition 5 , which can be found in [Lo]:

Definition 5 (Louveau). Let $X, Y$ be Polish spaces, $A$ a Borel subset of $X \times Y$ and $\Gamma$ a Baire (or Wadge) class. We say that $A$ is potentially in $\Gamma(\operatorname{denoted} A \in \operatorname{pot}(\Gamma))$ iff there is a finer Polish topology $\sigma$ (resp., $\tau$ ) on $X$ (resp., $Y$ ) such that $A$ is in $\Gamma([X, \sigma] \times[Y, \tau])$.

The previous result shows that this notion makes sense for product topologies. This notion is a natural invariant for $\leq_{B}$ : if $F$ is $\operatorname{pot}(\Gamma)$ and $E \leq_{B} F$, then $E$ is $\operatorname{pot}(\Gamma)$. Using this notion, A. Louveau showed that the collection of $\boldsymbol{\Sigma}_{\xi}^{0}$ equivalence relations is not cofinal for $\leq_{B}$, and deduced from this the non-existence of a maximum Borel equivalence relation for $\leq_{B}$ (this non-existence result is due to H. Friedman and L. Stanley). A. Louveau has also noticed more recently that one can associate a quasi-order relation $R_{A} \subseteq(X \times 2)^{2}$ to $A \subseteq X^{2}$ as follows:

$$
(x, i) R_{A}(y, j) \Leftrightarrow(x, i)=(y, j) \text { or }[(x, y) \in A \text { and }(i, j)=(0,1)] \text {. }
$$

Using this, one can see that, from the point of view of Borel reducibility, the study of Borel quasi-orders is essentially the study of arbitrary Borel subsets of the plane. This strengthens the motivation for studying arbitrary Borel subsets of the plane, from the point of view of potential complexity. We have a result concerning equivalence relations (see [H-K-Lo] :

Theorem 6 (Harrington-Kechris-Louveau). Let $X$ be a Polish space, E a Borel equivalence relation on $X$, and

$$
E_{0}:=\left\{(\alpha, \beta) \in 2^{\mathbb{N}} \times 2^{\mathbb{N}} / \exists n \in \mathbb{N} \quad \forall m \geq n \quad \alpha(m)=\beta(m)\right\} .
$$

Then exactly one of the following holds:

(a) The relation $E$ is $\operatorname{pot}\left(\boldsymbol{\Pi}_{1}^{0}\right)$.

(b) $E_{0} \leq_{B} E$ (with $u$ continuous and one-to-one).

We will study other structures than equivalence relations (for example quasiorders), and even arbitrary Borel subsets of the plane. We need some other notions 
of comparison. Let $X, Y, X^{\prime}, Y^{\prime}$ be Polish spaces, and $A$ (resp., $A^{\prime}$ ) a Borel subset of $X \times Y$ (resp., $X^{\prime} \times Y^{\prime}$ ). We set

$$
A \leq_{B}^{r} A^{\prime} \Leftrightarrow \exists u: X \rightarrow X^{\prime} \quad \exists v: Y \rightarrow Y^{\prime} \text { Borel with } A=(u \times v)^{-1}\left(A^{\prime}\right) .
$$

We want to extend the previous result to arbitrary Borel subsets of the plane. This works partially (see [L1]):

Theorem 7. Let $\Delta\left(2^{\mathbb{N}}\right):=\left\{(\alpha, \beta) \in 2^{\mathbb{N}} \times 2^{\mathbb{N}} / \alpha=\beta\right\}, L_{0}:=\left\{(\alpha, \beta) \in 2^{\mathbb{N}} \times 2^{\mathbb{N}} / \alpha<_{\operatorname{lex}} \beta\right\}$, $X, Y$ be Polish spaces, and $A$ a $\operatorname{pot}\left(\check{D}_{2}\left(\Sigma_{1}^{0}\right)\right)$ subset of $X \times Y$. Then exactly one of the following holds:

(a) The set $A$ is $\operatorname{pot}\left(\boldsymbol{\Pi}_{1}^{0}\right)$.

(b) $\neg \Delta\left(2^{\mathbb{N}}\right) \leq_{B}^{r} A$ or $L_{0} \leq_{B}^{r} A$ (with $u, v$ continuous and one-to-one).

Things become more complicated at the level $D_{2}\left(\boldsymbol{\Sigma}_{1}^{0}\right)$ (differences of two open sets; $\check{D}_{2}\left(\boldsymbol{\Sigma}_{1}^{0}\right)$ is the dual Wadge class of unions of a closed set and of an open set).

Theorem 8. The following statements are true:

(a) There is a perfect $\leq_{B}^{r}$-antichain $\left(A_{\alpha}\right)_{\alpha \in 2^{\mathbb{N}}} \subseteq D_{2}\left(\Sigma_{1}^{0}\right)\left(2^{\mathbb{N}} \times 2^{\mathbb{N}}\right)$ such that $A_{\alpha}$ is $\leq_{B}^{r}$-minimal among $\boldsymbol{\Delta}_{1}^{1} \backslash \operatorname{pot}\left(\boldsymbol{\Pi}_{1}^{0}\right)$ sets, for any $\alpha \in 2^{\mathbb{N}}$.

(b) There is a perfect $\leq_{B}$-antichain $\left(R_{\alpha}\right)_{\alpha \in 2^{\mathbb{N}}}$ such that $R_{\alpha}$ is $\leq_{B}$-minimal among $\boldsymbol{\Delta}_{1}^{1} \backslash \operatorname{pot}\left(\boldsymbol{\Pi}_{1}^{0}\right)$ sets, for any $\alpha \in 2^{\mathbb{N}}$. Moreover, $\left(R_{\alpha}\right)_{\alpha \in 2^{\mathbb{N}}}$ can be taken to be a subclass of any of the following classes:

- Graphs (i.e., irreflexive and symmetric relations).

- Oriented graphs (i.e., irreflexive and antisymmetric relations).

- Quasi-orders.

- Partial orders (i.e., reflexive, antisymmetric and transitive relations).

In other words, the case of equivalence relations, for which we have a unique (up to bi-reducibility) minimal non-potentially closed element with Theorem 6, is very specific. Theorem $8(\mathrm{~b})$ says, among other things, that the mixture between symmetry and transitivity is very strong.

Example. Let us specify the construction of the antichain in (a). For a set $C$ such that $C \subseteq 2^{<\mathbb{N}}$ we define

$$
A^{C}:=\left\{(s 0 \gamma, s 1 \gamma) / s \in C, \gamma \in 2^{\mathbb{N}}\right\} .
$$

For an infinite set $S$ such that $0 \in S \subseteq \mathbb{N}$ we define

$$
C_{S}:=\left\{t \in 2^{<\mathbb{N}} / \operatorname{Card}(t) \in S\right\},
$$

where $\operatorname{Card}(t)$ is the number of ones in $t$. Such an $S$ is always of the following form:

$$
S_{\beta}:=\left\{\Sigma_{i<j}(1+\beta(j)) / j \in \mathbb{N}\right\},
$$

where $\beta \in \mathbb{N}^{\mathbb{N}}$.

Theorem 9. The set $A^{C_{S}}$ is minimal for $\left[\boldsymbol{\Delta}_{1}^{1} \backslash \operatorname{pot}\left(\boldsymbol{\Pi}_{1}^{0}\right), \leq_{B}^{r}\right]$ if

$$
\forall p \in \mathbb{N} \quad \exists k \in \mathbb{N} \quad \forall q \in \mathbb{N} \quad \exists c \in \mathbb{N} \cap[q, q+k] \quad c+(S \cap[0, p])=S \cap(c+[0, p]) .
$$

It remains to define $\beta_{\alpha} \in 2^{\mathbb{N}} \subseteq \mathbb{N}^{\mathbb{N}}$, for $\alpha \in 2^{\mathbb{N}}$. We inductively define a sequence $\left(s_{\alpha, n}\right)_{n} \subseteq 2^{<\mathbb{N}}$ as follows: $s_{\alpha, 0}:=0, s_{\alpha, 1}:=1, s_{\alpha, n+2}:=s_{\alpha, n}^{\alpha(n)+1} s_{\alpha, n+1}^{\alpha(n+1)+1}$. Note that $s_{\alpha, n} \prec \neq s_{\alpha, n+2}$, so that we can define $\beta_{\alpha}:=\lim _{n \rightarrow \infty} s_{\alpha, 2 n} \in 2^{\mathbb{N}}$. It is suitable: $\left(A^{C_{S_{\beta_{\alpha}}}}\right)_{\alpha \in 2^{\mathrm{N}}}$ is a perfect antichain made of minimal sets for $\left[\boldsymbol{\Delta}_{1}^{1} \backslash \operatorname{pot}\left(\boldsymbol{\Pi}_{1}^{0}\right), \leq_{B}^{r}\right]$. 


\section{REDUCTION BY HOMOMORPHISM}

Theorem 8(a) shows that the classical notions of reduction (on the whole product) do not work, at least at the first level. So we must find another notion of comparison. We have a positive result with another notion, which is in some sense "half of the Borel reducibility ordering". Let $A$ (resp., $A^{\prime}$ ) be an analytic subset of $X \times X\left(\right.$ resp.,$\left.X^{\prime} \times X^{\prime}\right)$. We set

$$
(X, A) \preceq_{B}\left(X^{\prime}, A^{\prime}\right) \Leftrightarrow \exists u: X \rightarrow X^{\prime} \text { Borel with } A \subseteq(u \times u)^{-1}\left(A^{\prime}\right) .
$$

This notion essentially makes sense for irreflexive relations (we can take $u$ to be constant if $A^{\prime}$ is not irreflexive).

Notation. Let $\psi: \mathbb{N} \rightarrow 2^{<\mathbb{N}}$ be the natural bijection $(\psi(0)=\emptyset, \psi(1)=0, \psi(2)=1$, $\left.\psi(3)=0^{2}, \psi(4)=01, \psi(5)=10, \psi(6)=1^{2}, \ldots\right)$. Note that $|\psi(n)| \leq n$, so that we can define $s_{n}:=\psi(n) 0^{n-|\psi(n)|}$. The crucial properties of $\left(s_{n}\right)$ are that it is dense (there is $n$ such that $t \prec s_{n}$, for each $\left.t \in 2^{<\omega}\right)$, and that $\left|s_{n}\right|=n$. We put

$$
A_{0}:=A^{\left\{s_{n} / n \in \mathbb{N}\right\}}=\left\{\left(s_{n} 0 \gamma, s_{n} 1 \gamma\right) / n \in \mathbb{N}, \gamma \in 2^{\mathbb{N}}\right\} .
$$

The symmetric set $s\left(A_{0}\right)$ generated by $A_{0}$ is considered in [K-S-T], where the following is essentially shown:

Theorem 10 (Kechris, Solecki, Todorčević). Let $X$ be a Polish space, and $A$ an analytic subset of $X \times X$. Then exactly one of the following holds:

(a) $(X, A) \preceq_{B}(\mathbb{N}, \neq)$.

(b) $\left(2^{\mathbb{N}}, A_{0}\right) \preceq_{B}(X, A)$ (with u continuous).

In [K-S-T], it is conjectured that we can have $u$ one-to-one in Theorem 10(b). This is not the case.

\section{Reduction on a Closed SET}

As a consequence of Theorem 10, we have the following:

Theorem 11. Let $X, Y$ be Polish spaces, and $A$ a Borel subset of $X \times Y$. Then exactly one of the following holds:

(a) The set $A$ is $\operatorname{pot}\left(\boldsymbol{\Pi}_{1}^{0}\right)$.

(b) There are $u: 2^{\mathbb{N}} \rightarrow X$ and $v: 2^{\mathbb{N}} \rightarrow Y$ continuous with $A_{0}=(u \times v)^{-1}(A) \cap \overline{A_{0}}$. Moreover, we can neither ensure that $u$ and $v$ are one-to-one, nor remove $\overline{A_{0}}$.

So we get a minimum non-potentially closed set if we do not ask for a reduction on the whole product. To generalize Theorem 11, the right way to see $A_{0}$ seems to be the following. Let $T_{0}$ be the tree associated with $\overline{A_{0}}=A_{0} \cup \Delta\left(2^{\mathbb{N}}\right)$ :

$$
T_{0}=\left\{(s, t) \in 2^{<\mathbb{N}} \times 2^{<\mathbb{N}} / s=t \text { or } \exists n \in \mathbb{N} \exists w \in 2^{<\mathbb{N}}(s, t)=\left(s_{n} 0 w, s_{n} 1 w\right)\right\} .
$$

The map $\Delta: 2^{\mathbb{N}} \times 2^{\mathbb{N}} \rightarrow 2^{\mathbb{N}}$ is the symmetric difference: $\Delta(\alpha, \beta)(i):=(\alpha \Delta \beta)(i)=1$ exactly when $\alpha(i) \neq \beta(i)$, for $i \in \mathbb{N}$. Let $S_{1}:=\left\{\gamma \in 2^{\mathbb{N}} / \exists i \in \mathbb{N} \gamma(i)=1\right\}$ be the typical one-dimensional $\boldsymbol{\Sigma}_{1}^{0} \backslash \boldsymbol{\Pi}_{1}^{0}$ set. We have

$$
A_{0}=\left\{(\alpha, \beta) \in 2^{\mathbb{N}} \times 2^{\mathbb{N}} /(\alpha, \beta) \in\left[T_{0}\right] \text { and } \alpha \Delta \beta \in S_{1}\right\} .
$$

This scheme can be generalized. Theorem 11 shows that we cannot have only one minimal non-potentially closed set, for the reduction on the whole product. The reduction is possible on a closed set (the closure of $A_{0}$ ). This closure does not 
explain why we cannot have a reduction on the whole product. This comes from Theorem 8. The orthogonality between the examples appearing in the antichains of its statement comes from different types of cycles. This will give a better explanation than the closure. We will replace the closure with a closed set, which will be seen as the set of branches of some tree on $2 \times 2$. This tree will have the acyclicity properties that we need. This leads to the following definition:

Definition 12. Let $R$ be a relation on a set $E$.

- An $R$-path is a finite sequence $\left(e_{i}\right)_{i \leq n} \subseteq E$ such that $\left(e_{i}, e_{i+1}\right) \in R$, for $i<n$.

- An $\overline{R \text {-cycle }}$ is an $R$-path $\left(e_{i}\right)_{i \leq n}$ such that $n \geq 3$ and

$$
\left[0 \leq i \neq j \leq n \text { and } e_{i}=e_{j}\right] \Leftrightarrow\{i, j\}=\{0, n\} .
$$

- We say that $R$ is acyclic if there is no $R$-cycle.

- We say that a tree $\overline{T \text { on }} 2 \times 2$ is uniformly acyclic if, for each $p>0$,

(a) The relation $T \cap\left(2^{p} \times 2^{p}\right)$ is irreflexive and antisymmetric.

(b) The symmetric relation $s\left(T \cap\left(2^{p} \times 2^{p}\right)\right)$ generated by $T \cap\left(2^{p} \times 2^{p}\right)$ is acyclic.

The main new results in this paper are the following:

Theorem 13 (Debs-Lecomte). Let $T$ be a uniformly acyclic tree, $\xi<\aleph_{1}, A_{1+\xi}$ in $\Sigma_{1+\xi}^{0}([T]), X, Y$ Polish spaces, and $A, B$ disjoint analytic subsets of $X \times Y$. Then one of the following holds:

(a) The set $A$ is separable from $B$ by a $\operatorname{pot}\left(\mathbf{\Pi}_{1+\xi}^{0}\right)$ set.

(b) There are $u: 2^{\mathbb{N}} \rightarrow X$ and $v: 2^{\mathbb{N}} \rightarrow Y$ continuous with $A_{1+\xi} \subseteq(u \times v)^{-1}(A)$ and $[T] \backslash A_{1+\xi} \subseteq(u \times v)^{-1}(B)$.

This is a dichotomy if we moreover assume that $A_{1+\xi} \notin \operatorname{pot}\left(\mathbf{\Pi}_{1+\xi}^{0}\right)$.

This result has initially been proved by D. Lecomte when $1+\xi$ is a successor ordinal. Then G. Debs proved it when $1+\xi$ is a limit ordinal. The proof of Theorem 13 uses the representation theorem for Borel sets in D-SR. Note that we can deduce Theorem 2 from the proof of Theorem 13. Theorem 13 is the analog of Theorem 2 in dimension two (see [Lo-SR, also Theorem III-2.1 in [D-SR]). The tree $T$ has to be small enough, since there is no possibility to have a reduction on the whole product. But at the same time, $T$ has to be big enough to ensure the existence of complicated sets inside $[T]$ :

Theorem 14. There are concrete examples of:

(a) A uniformly acyclic tree $T$.

(b) $A$ set $A_{1+\xi} \in \boldsymbol{\Sigma}_{1+\xi}^{0}([T]) \backslash \operatorname{pot}\left(\boldsymbol{\Pi}_{1+\xi}^{0}\right)$, for $\xi<\aleph_{1}$.

This result is the complement of Theorem 13 (which is true with $T:=\emptyset !$ ). Again, the couple Theorems $13-14$ is the analog of the couple Theorems 2-3.

\section{The EXAMPles}

Let us specify the examples of Theorem 14 . Let $\varphi=\left(\varphi_{0}, \varphi_{1}\right): \mathbb{N} \rightarrow \mathbb{N}^{2}$ be the natural bijection. More precisely, we set, for $q \in \mathbb{N}$,

$$
M(q):=\max \left\{m \in \mathbb{N} / \Sigma_{k \leq m} k \leq q\right\} .
$$


Then we define $\varphi(q)=\left(\varphi_{0}(q), \varphi_{1}(q)\right):=\left(M(q)-q+\left(\Sigma_{k \leq M(q)} k\right), q-\left(\Sigma_{k \leq M(q)} k\right)\right)$. One can check that $\langle i, j\rangle:=\varphi^{-1}(i, j)=\left(\Sigma_{k \leq i+j} k\right)+j$. More concretely, we get

$$
\varphi[\mathbb{N}]=\{(0,0) ;(1,0) ;(0,1) ;(2,0) ;(1,1) ;(0,2) ; \ldots\} .
$$

Definition 15. We say that $E \subseteq \bigcup_{q \in \mathbb{N}} 2^{q} \times 2^{q}$ is a test if

(a) $\forall q \in \mathbb{N} \quad \exists$ ! $\left(s_{q}, t_{q}\right) \in E \cap\left(2^{q} \times 2^{q}\right)$.

(b) $\forall m, q \in \mathbb{N} \quad \forall u \in 2^{<\mathbb{N}} \exists v \in 2^{<\mathbb{N}}\left(s_{q} 0 u v, t_{q} 1 u v\right) \in E$ and $\varphi_{0}\left(\left|t_{q} 1 u v\right|-1\right)=m$.

(c) $\forall n>0 \quad \exists q<n \quad \exists w \in 2^{<\mathbb{N}} \quad s_{n}=s_{q} 0 w$ and $t_{n}=t_{q} 1 w$.

We will call $T$ the tree generated by a test $E=\left\{\left(s_{q}, t_{q}\right) / q \in \mathbb{N}\right\}$ :

$$
T:=\left\{(s, t) \in 2^{<\mathbb{N}} \times 2^{<\mathbb{N}} / s=t=\emptyset \text { or } \exists q \in \mathbb{N} \quad \exists w \in 2^{<\mathbb{N}} s=s_{q} 0 w \text { and } t=t_{q} 1 w\right\} .
$$

One can show the existence of a test, and that $T$ is uniformly acyclic. The uniqueness condition in (a) and condition (c) ensure that $T$ is small enough, and also the acyclicity. The existence condition in (a) and condition (b) ensure that $T$ is big enough. More specifically, if $X$ is a Polish space and $\sigma$ a finer Polish topology on $X$, then there is a dense $G_{\delta}$ subset of $X$ on which the two topologies coincide. The first part of condition (b) ensures the possibility to get inside the square of a dense $G_{\delta}$ subset of $2^{\omega}$. The examples of Theorem 14(b) are constructed using the examples in [Lo-SR]. Conditions on the verticals appear, and the second part of condition (b) gives control of the choice of verticals.

Notation. In [Lo-SR, Lemma 3.3, the map $\rho_{0}: 2^{\mathbb{N}} \rightarrow 2^{\mathbb{N}}$ is introduced as follows:

$$
\rho_{0}(\varepsilon)(i):=\left\{\begin{array}{l}
1 \text { if } \varepsilon(\langle i, j\rangle)=0, \text { for each } j \in \mathbb{N}, \\
0 \text { otherwise. }
\end{array}\right.
$$

In that paper, $\rho_{0}^{\xi}: 2^{\mathbb{N}} \rightarrow 2^{\mathbb{N}}$ is also defined for $\xi<\aleph_{1}$, by induction on $\xi$ (see the proof of Theorem 3.2), as follows. We put $\rho_{0}^{0}:=\operatorname{Id}_{2^{\mathbb{N}}}, \rho_{0}^{\eta+1}:=\rho_{0} \circ \rho_{0}^{\eta}$. If $\lambda>0$ is limit, then fix $\left(\xi_{k}^{\lambda}\right) \subseteq \lambda \backslash\{0\}$ such that $\Sigma_{k} \xi_{k}^{\lambda}=\lambda$. For $\varepsilon \in 2^{\mathbb{N}}$ and $k \in \mathbb{N}$, we define $(\varepsilon)^{k} \in 2^{\mathbb{N}}$ by $(\varepsilon)^{k}(i):=\varepsilon(i+k)$. We also define $\rho_{0}^{(k, k+1)}: 2^{\mathbb{N}} \rightarrow 2^{\mathbb{N}}$ by

$$
\rho_{0}^{(k, k+1)}(\varepsilon)(i):=\left\{\begin{array}{l}
\varepsilon(i) \text { if } i<k, \\
\rho_{0}^{\xi_{k}^{\lambda}}\left((\varepsilon)^{k}\right)(i-k) \text { if } i \geq k .
\end{array}\right.
$$

We set $\rho_{0}^{(0, k+1)}:=\rho_{0}^{(k, k+1)} \circ \rho_{0}^{(k-1, k)} \circ \cdots \circ \rho_{0}^{(0,1)}$ and $\rho_{0}^{\lambda}(\varepsilon)(k):=\rho_{0}^{(0, k+1)}(\varepsilon)(k)$.

The set $H_{1+\xi}:=\left(\rho_{0}^{\xi}\right)^{-1}\left(\left\{0^{\infty}\right\}\right)$ is also introduced, and the authors show that $H_{1+\xi}$ is $\Pi_{1+\xi}^{0} \backslash \boldsymbol{\Sigma}_{1+\xi}^{0}$ (see Theorem 3.2).

- The map $S: 2^{\mathbb{N}} \rightarrow 2^{\mathbb{N}}$ is the shift map: $S(\alpha)(i):=\alpha(i+1)$.

- Let $T$ be the tree generated by a test. We put, for $\xi<\aleph_{1}$,

$$
A_{1+\xi}:=\left\{(\alpha, \beta) \in 2^{\mathbb{N}} \times 2^{\mathbb{N}} /(\alpha, \beta) \in[T] \text { and } S(\alpha \Delta \beta) \notin H_{1+\xi}\right\} .
$$

Then $A_{1+\xi}$ is $\boldsymbol{\Sigma}_{1+\xi}^{0}([T]) \backslash \operatorname{pot}\left(\boldsymbol{\Pi}_{1+\xi}^{0}\right)$. We now introduce a notation to state the crucial lemma used to show it.

Notation. We define $p: \mathbb{N}<\mathbb{N} \backslash\{\emptyset\} \rightarrow \mathbb{N}$. We actually define $p(s)$ by induction on $|s|$ :

$$
p(s):=\left\{\begin{array}{l}
s(0) \text { if }|s|=1, \\
\langle p(s\lceil(|s|-1)), s(|s|-1)\rangle \text { otherwise. }
\end{array}\right.
$$

Note that $\left.p\right|_{\mathbb{N}^{n}}: \mathbb{N}^{n} \rightarrow \mathbb{N}$ is a bijection for each $n \geq 1$. 
Lemma 16. Let $G$ be a dense $G_{\delta}$ subset of $2^{\mathbb{N}}$. Then there is $\alpha_{0} \in G$ and a continuous $f: 2^{\mathbb{N}} \rightarrow G$ such that, for each $\alpha \in 2^{\mathbb{N}}$,

(a) $\left(\alpha_{0}, f(\alpha)\right) \in[T]$.

(b) For each $t \in \mathbb{N}<\mathbb{N}$, and each $m \in \mathbb{N}$,

(i) $\alpha(p(t m))=1 \Rightarrow \exists m^{\prime} \in \mathbb{N}\left(\alpha_{0} \Delta f(\alpha)\right)\left(p\left(t m^{\prime}\right)+1\right)=1$.

(ii) $\left(\alpha_{0} \Delta f(\alpha)\right)(p(t m)+1)=1 \Rightarrow \exists m^{\prime} \in \mathbb{N} \quad \alpha\left(p\left(t m^{\prime}\right)\right)=1$.

\section{Complements of the main Results}

Now we come to consequences of Theorems 13 and 14. To state them, we need more notation. We use some tools from effective descriptive set theory (the reader should see $[\mathrm{M}]$ for basic notions about it).

Notation. Let $X$ be a recursively presented Polish space. We denote by $\Delta_{X}$ the topology on $X$ generated by $\Delta_{1}^{1}(X)$. This topology is Polish (see the proof of Theorem $3.4 \mathrm{in}[\mathrm{Lo}]$ ). We set $\tau_{1}:=\Delta_{X} \times \Delta_{Y}$ if $Y$ is also a recursively presented Polish space.

- Let $2 \leq \xi<\omega_{1}^{\mathrm{CK}}$. The topology $\tau_{\xi}$ is generated by $\Sigma_{1}^{1}(X \times Y) \cap \boldsymbol{\Pi}_{<\xi}^{0}\left(\tau_{1}\right)$. Note that $\boldsymbol{\Sigma}_{1}^{0}\left(\tau_{\xi}\right) \subseteq \boldsymbol{\Sigma}_{\xi}^{0}\left(\tau_{1}\right)$, so that $\boldsymbol{\Pi}_{1}^{0}\left(\tau_{\xi}\right) \subseteq \boldsymbol{\Pi}_{\xi}^{0}\left(\tau_{1}\right)$.

- Recall the existence of $\Pi_{1}^{1}$ sets $W^{X} \subseteq \mathbb{N}, C^{X} \subseteq \mathbb{N} \times X$ such that $\Delta_{1}^{1}(X)=$ $\left\{C_{n}^{X} / n \in W^{X}\right\}, \quad\left\{(n, x) \in \mathbb{N} \times X / n \in W^{X}\right.$ and $\left.x \notin C_{n}^{X}\right\} \in \Pi_{1}^{1}(\mathbb{N} \times X)$ (see [H-K-Lo, Theorem 3.3.1).

- Set $\operatorname{pot}\left(\boldsymbol{\Pi}_{0}^{0}\right):=\boldsymbol{\Delta}_{1}^{1}(X) \times \boldsymbol{\Delta}_{1}^{1}(Y)$ and, for $\xi<\omega_{1}^{\mathrm{CK}}$,

$$
W_{\xi}^{X \times Y}:=\left\{p \in W^{X \times Y} / C_{p}^{X \times Y} \in \operatorname{pot}\left(\boldsymbol{\Pi}_{\xi}^{0}\right)\right\} .
$$

We also set $W_{<\xi}^{X \times Y}:=\bigcup_{\eta<\xi} W_{\eta}^{X \times Y}$.

Theorem 17 (Debs-Lecomte-Louveau). Let $T$ be given by Theorem $14, \xi<\omega_{1}^{\mathrm{CK}}$, $A_{1+\xi}$ be given by Theorem 14, and $X, Y$ be recursively presented Polish spaces.

(1) Let $A, B$ be disjoint $\Sigma_{1}^{1}$ subsets of $X \times Y$. The following are equivalent:

(a) The set $A$ cannot be separated from $B$ by a $\operatorname{pot}\left(\boldsymbol{\Pi}_{1+\xi}^{0}\right)$ set.

(b) The set $A$ cannot be separated from $B$ by a $\Delta_{1}^{1} \cap \operatorname{pot}\left(\mathbf{\Pi}_{1+\xi}^{0}\right)$ set.

(c) The set $A$ cannot be separated from $B$ by a $\boldsymbol{\Pi}_{1+\xi}^{0}\left(\tau_{1}\right)$ set.

(d) $\bar{A}^{\tau_{1}+\xi} \cap B \neq \emptyset$.

(e) There are continuous $u: 2^{\mathbb{N}} \rightarrow X$ and $v: 2^{\mathbb{N}} \rightarrow Y$ with $A_{1+\xi} \subseteq(u \times v)^{-1}(A)$ and $[T] \backslash A_{1+\xi} \subseteq(u \times v)^{-1}(B)$.

(2) The sets $W_{1+\xi}^{X \times Y}$ and $W_{<1+\xi}^{X \times Y}$ are $\Pi_{1}^{1}$.

The equivalence between (a), (b) and (c), and also (2), is shown in Lo. We can assume this equivalence and (2), then show Theorems 13, 14, and then prove Theorem 17 . We can also directly prove Theorem 17 by induction on $\xi$. An immediate consequence of this is the following, shown in $[\mathrm{LO}$ :

Corollary 18 (Louveau). Let $\xi<\omega_{1}^{\mathrm{CK}}, X, Y$ be recursively presented Polish spaces, and $A$ a $\Delta_{1}^{1}$ subset of $X \times Y$. The following are equivalent:

(a) The set $A$ is $\operatorname{pot}\left(\boldsymbol{\Pi}_{1+\xi}^{0}\right)$.

(b) The set $A$ is $\mathbf{\Pi}_{1+\xi}^{0}\left(\tau_{1}\right)$.

We also have the following consequence of Theorems 13 and 14: 
Corollary 19 (Debs-Lecomte). Let $\xi<\aleph_{1}$. There is a Borel subset $A_{1+\xi}$ of $2^{\mathbb{N}} \times 2^{\mathbb{N}}$ such that for any Polish spaces $X, Y$, and for any disjoint analytic subsets $A, B$ of $X \times Y$, exactly one of the following holds:

(a) The set $A$ is separable from $B$ by a $\operatorname{pot}\left(\mathbf{\Pi}_{1+\xi}^{0}\right)$ set.

(b) There are continuous $u: 2^{\mathbb{N}} \rightarrow X$ and $v: 2^{\mathbb{N}} \rightarrow Y$ with $A_{1+\xi} \subseteq(u \times v)^{-1}(A)$ and $\overline{A_{1+\xi}} \backslash A_{1+\xi} \subseteq(u \times v)^{-1}(B)$.

Moreover we can neither ensure that $u$ and $v$ are one-to-one if $\xi \leq 1$, nor replace $\overline{A_{1+\xi}} \backslash A_{1+\xi}$ with $\left(2^{\mathbb{N}} \times 2^{\mathbb{N}}\right) \backslash A_{1+\xi}$.

The one-to-one complement is due to D. Lecomte (see Theorem 11 when $\xi=0$, and Theorem 15 in L2 when $\xi=1$ ). The latter complement has initially been shown by D. Lecomte when $\xi \leq 1$ (see, for example, Theorem 11). Then G. Debs found a simpler proof, which moreover works in the general case.

\section{REFERENCES}

[D-SR] G. Debs and J. Saint Raymond, Borel liftings of Borel sets: some decidable and undecidable statements, to appear in Memoirs of the Amer. Math. Soc.

[H-K-Lo] L. A. Harrington, A. S. Kechris, and A. Louveau, A Glimm-Effros dichotomy for Borel equivalence relations, J. Amer. Math. Soc. 3 (1990), 903-928. MR1057041(91h:28023)

[K] A. S. Kechris, Classical Descriptive Set Theory, Springer-Verlag, 1995. MR.1321597 (96e:03057)

[K-S-T] A. S. Kechris, S. Solecki, and S. Todorčević, Borel chromatic numbers, Adv. Math. 141 (1999), 1-44. MR 1667145 (2000e:03132)

[L1] D. Lecomte, Classes de Wadge potentielles et théorèmes d'uniformisation partielle, Fund. Math. 143 (1993), 231-258. MR1247803 (94i:03099)

[L2] D. Lecomte, Complexité des boréliens à coupes dénombrables, Fund. Math. 165 (2000), 139-174. MR 1808728 (2001k:54061)

[Lo] A. Louveau, Ensembles analytiques et boréliens dans les espaces produit, Astérisque (S. M. F.) 78 (1980). MR0606933(82j:03062)

[M] Y. N. Moschovakis, Descriptive Set Theory, North-Holland, 1980. MR0561709 (82e:03002)

[Lo-SR] A. Louveau and J. Saint Raymond, Borel classes and closed games: Wadge-type and Hurewicz-like results, Trans. Amer. Math. Soc. 304 (1987), 431-467. MR0911079 (89g:03068)

[SR] J. Saint Raymond, La structure borélienne d'Effros est-elle standard?, Fund. Math. 100 (1978), 201-210. MR0509546 (80g:54044)

Université Paris 6, Equipe d'Analyse Fonctionnelle, tour 46-0, Boîte 186, 4, place Jussieu, 75252 Paris Cedex 05, France, and Université de Picardie, I.U.T. de l'Oise, site de Creil, 13, allée de la faïencerie, 60107 Creil, France

E-mail address: lecomte@moka.ccr.jussieu.fr 